\title{
The Role of Color in Healthcare Environments, Emergent Bodies of Evidence-based Design Approach
}

\author{
Hessam Ghamari ${ }^{1, *}$, Cherif Amor ${ }^{2}$ \\ ${ }^{1}$ Department of Applied Design, Appalachian State University, United States \\ ${ }^{2}$ Department of Interior Design, Virginia Commonwealth University in Qatar, Qatar
}

Copyright $\mathrm{O} 2016$ by authors, all rights reserved. Authors agree that this article remains permanently open access under the terms of the Creative Commons Attribution License 4.0 International License

\begin{abstract}
The objective of this study is to overview the literature relative to color, as an environmental constituent, and its impacts in healthcare environments. Borrowing from the environmental behavioral paradigm, this study attempts to decipher myths and misconceptions as well as highlight well-evidenced research findings. Broad Literature review journal articles and reports of empirical studies in multiple disciplines were studied to identify theories, which could have design implications for color in healthcare design. Despite the incongruity and fragmentation of previous studies, it emerges from this overview that color impacts healthcare outcomes by reducing medical errors, promoting the sense of well-being, reducing stress, improving patients sleep, reducing length of stay, reducing spatial disorientation, increasing patient satisfaction, and increasing staff morale and productivity. While the review of literature indicates major findings relative to the impact of color on healthcare environments, yet ambiguities remained to be addressed. Previous studies on the use of color in healthcare environment have illustrated that there are some obvious, replicable, behavioral and perceptual effects from color that addressed their use in certain ways for design. However, color must be observed and analyzed in the contextual application to avoid generalizations about color perception and mood affects.
\end{abstract}

Keywords Color, Patient, Health, Well-being, Evidence Based Design

\section{Introduction}

Color is a fundamental factor in environmental design. People often consider our response to color as instinctive and simple, but it involves a very complex interaction between light, eyes, and the brain. The role of color is important not only because it influences aesthetic and technical aspects of human-made environments, but also it affects human beings psychologically, physiologically [1]. Several studies have been conducted on the significance of color in healthcare environments by reducing stress, medical errors, depression, length of stay, improving social support of patients, communications in healthcare environment, circadian rhythms, patients sleep, improving satisfaction, and increasing staff productivity and morale [1,2]. Although there is a reasonable body of knowledge on the effects of color on emotions, a lack of research is sensed, especially in healthcare settings [3].

Based on an evidence-based design perspective, this study reviewed the impact of color on outcomes in healthcare settings using a growing body of publications, including but not limited to design and allied disciplines. To do so, the nature of color-theories and perceptions - as well as psychological effects of color were reviewed. Looking at the perception of color, different characteristics of light sources, human responses to light and color physiologically and psychologically, and individual differences were considered. Next, the significance of color in healthcare environment was described and different areas in which color can impact the healthcare outcomes were indicated. According to the precedents, color contribute to improve the outcomes in healthcare environment by reducing medical errors, promoting the sense of well-being, reducing stress, improving the patient sleep and circadian rhythm, reducing length of stay, improving spatial orientation and way finding, increasing patient satisfaction, and increasing staff morale and productivity.

The U.S. healthcare system is involved with the critical need of replacing aging 1970s hospitals. Moreover, population shifts, the graying of the baby boom generation, and the introduction of new medical technologies are other reasons that make the consideration of healthcare interior design environments urgent. It is expected in the next five years, the United States will spend more than $\$ 180$ billion for new hospitals. These new hospitals will be used in place for decades [2]. Additionally, It was found that hospital visitors experience emotional circumstances and their concentration can be clouded by anxiety [4]. Problems with healthcare system not only negatively influence patients; but also affect 
staff [2].

The body of knowledge on color application, however, has been fragmented, debatable, and anecdotal. Many healthcare designers and practitioners have questioned the relationships between color and people, suspected that the psychotherapeutic effects of color, and searched for empirical reasoning for the different color guidelines in healthcare settings [1]. This research aims to identify research findings commonalities and differences and provide recommendations for future research endeavors. To do so, this study will examine color impact on healthcare by deciphering misconceptions and underscoring well-evidenced applications.

\subsection{Nature of Color}

Nassau [5] indicated that color is a part of perception process in which it is carried to us from our surroundings according to the wavelengths differences of light. Color is perceived by the eye, and interpreted by the brain. The perception of color is a complicated process that involves light source attributes, surfaces' texture, and neurological interpretations. Light reflects off surfaces and transmits an electrochemical response into the eye, which translates into color within the brain [6]. Different colored surfaces are separated by a different pattern of neural signals that are generated by color receptors found within the retina of the eye $[7,8]$. The perception of color depends on the light source characteristics such as the Spectral Energy Distribution (SED), Color Rendering Index (CRI), and color temperature; materials characteristics such as the Light Reflectance Value (LRV); and space users' psychological and physiological characteristics including but not limited to culture, age, and visual acuity [1,5]. The use of different light sources in healthcare environments suggests a thorough understanding of the context, application, and user's specifics to facilitate appropriate visualization and interpretation of color perception and effects. Color perception is further influenced by the interaction of the intrinsic attributes of color - color hue, saturation, and intensity, and interaction with Light Reflectance Values LRV - but also the light sources attributes: Special Energy Distribution, Color Rendering Index, and Color Temperature. Spectral.

\subsubsection{Energy Distribution (SED)}

"SED is the amount of power from the light source in each color band or spectral region." This explains in part the difference between the light that comes from a standard cool-white [CW] fluorescent lamp, natural light, and the way it changes the colors of objects that reflect [1]. Figure 1 differentiates between characteristic of spectral energy distributions (SEDs) for an incandescent lamp (left) and a fluorescent lamp (right). The indicated Spectral Energy Distribution attribute of the light sources is a good indication of the color theme outcome, fluctuating between cool—violet, blue, and green - compared to warm theme including yellow, orange and red hues. Figure 1 illustrates the color warm theme generated through an incandescent light source in the range of 550 nanometers and above, while the cool color theme generated through a fluorescent source in the range of 400-550 nanometers.

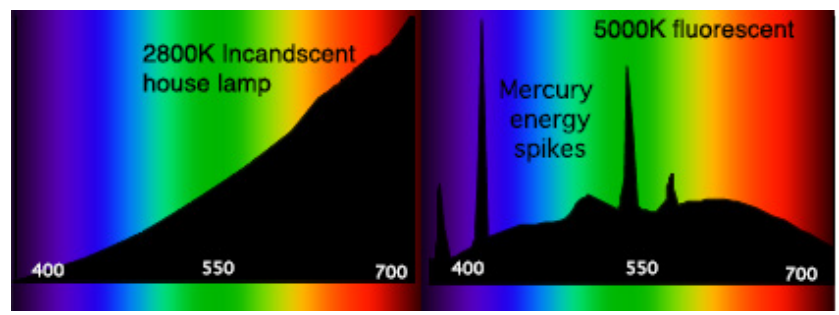

Figure 1. Characteristics spectral energy distributions (SEDs) for an incandescent lamp (left) and a fluorescent lamp (right)

\subsubsection{Color Rendering Index (CRI)}

International Electro technical Commission defined (IEC) has defined CRI as "a measure of the degree of color shift objects undergo when illuminated by the light source as compared with those same objects when illuminated by a reference source of comparable color temperature ". CRI is an indication of how well colors are rendered under a given light source and depends on the lamps' spectral power distribution. In comparison to daylight, the artificial light sources have a system of measuring color to describe the manner color are rendered. It is assumed that a maximum value equal to 100 corresponds to the color rendering of an incandescent lamp. Light sources with poor spectral content are characterized by a low value of CRI.

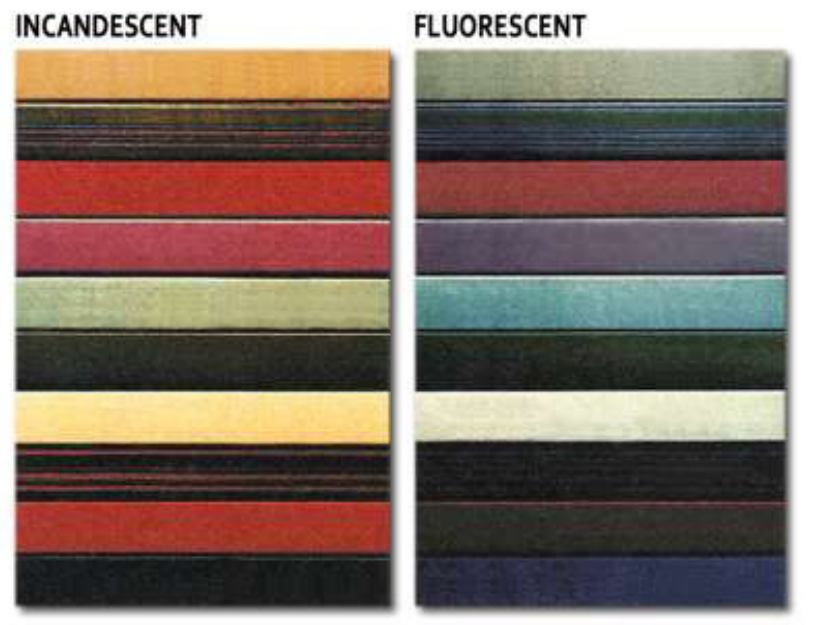

Figure 2. Emitted light spectrum determines CRI

\subsubsection{Color Temperature}

Color temperature of a light source is the absolute temperature of a blackbody radiator having a chromaticity equal to that of the light source (IESNA). Color of temperature is measured in Kelvin degrees. For example, a candle flame has a color temperature of about $2000 \mathrm{~K}$, incandescent lamp $2700 \mathrm{~K}$ and white halogen lamps typically have a color temperature of $2900 \mathrm{~K}$. Light sources 
color temperature is another important influencing color attribute that should be taken into account for instance the same wattage and lumen output might generate different feelings such as the case with fluorescent light sources: a) Warm White WW with a $2700 \mathrm{CCT}$, b) Cool White CW with a 4100 CCT, and c) Daylight DX with 5500 CT). Illustratively, figure 1 illustrates how an incandescent light source - with a color temperature of $3000^{\circ} \mathrm{K}$ and a CRI of 99-augment warm colors and reduces dulls cool colors, while a cool fluorescent lamp-with a correlated color temperature of $4400^{\circ} \mathrm{K}$ and a CRI of 67 - enhances blue and attenuates red [9].

\subsubsection{Light Reflectance Value (LRV)}

Light reflectance value is the total quantity of visible and useable light reflected by a surface in all directions and at all wavelengths when illuminated by a light source. LRV is a measurement that indicates how much light a color reflects, and conversely how much it absorbs.

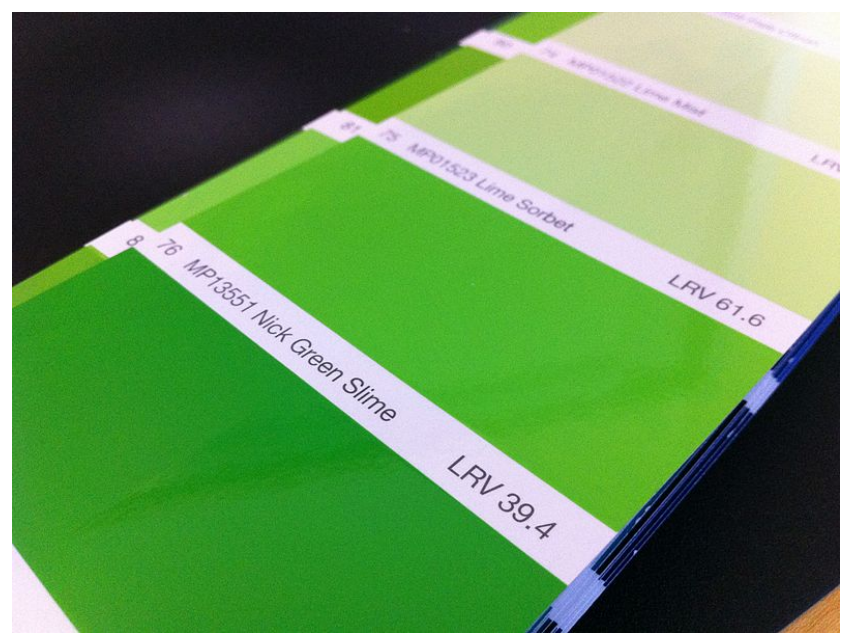

Figure 3. Light reflectance value of different surfaces

\subsection{Human Responses to Color}

Color, as one of the most important elements in our physical environment, has a great impact on our psychological and physiological responses [10]. Color and lighting can have an impact on peoples' perceptions and responses to the environment [4,11]. Michael Hemphill [12] showed that colors appear to elicit certain emotional responses. Birren [11] suggested that a clear and universal causal relationship exists between color and human response, and he cited Goldstein's assertion that "It is probably not a false statement if we say that a specific color stimulation is accompanied by a specific response pattern of the entire (human) organism"' (p. 144). By contrast, Wise [13] posited that previous studies found that there is still no established empirical evidence on the power of color. In fact it was found that the research failed to establish that any single color can affect our bodies and emotions in long term. However, color has been found to have a physical effect, certainly for short periods [14]. A research conducted in University of Lund showed that a warm color and a cool color have different psychological meanings, e.g., in the sense that they activate different associational themes [15]. In addition, another study indicated that although warm and cool colors have opposite effects on human minds, combining them can create comfortable atmospheres and settings [16].

\subsubsection{Biological Effects}

In 1976, Rikard Kuller explained how color and visual patterning affects not only the cortex, but also the entire central nervous system. Additionally, it has been found that when color is transmitted through the human eye, the brain releases the hypothalamus hormone, which affects our moods, mental clarity and energy level [17]. Gerard [18] investigated the effects of different colored screens projected by red, blue, and white light. Twenty-four males' blood pressure, palmary conductance, respiration rate, heart rate, eye blink frequency, and EEG were measured while they were exposed to each light for a total of ten minutes. He found statistical differences between the responses to red and blue lights for all physiological measures except heart rate. In addition, Gerard [18] argued that red light was more arousing than blue light on visual cortical activity and functions of the autonomic nervous system. He found that red increased blood pressure, respiration, and frequency of eye blink, whereas blue had the opposite effect. He proposed that responses to colors are predictable because they result in changes to the whole organism. Erwin, Lerner, Wilson, and Wilson [19] investigated arousal level by measuring suppression of alpha waves. Subjects were exposed to red, blue, green, and yellow colors for five minutes each. They found that the duration of alpha wave onset was shortest for the green, but they could not find a significant difference in arousal among others. Ali [20] investigated patterns of EEG recovery under red and blue lights. Subjects were exposed to red or blue light for either 5 or 10 minutes. He found more arousal when his subjects were exposed to red light than blue light.

Fehrman and Fehrman [21] reported on the effects of blue, red, and yellow on task performance and arousal for the effective color selection of interior spaces. The saturation and brightness of the colors were precisely controlled in order to demonstrate the effect of hue. Forty-two subjects performed mathematical exercises, reading, and motor activity tasks in colored rooms while their galvanic skin response (GSR) and pulse rate were measured. The researchers found that those who experienced the colors of equal saturation and brightness had comparable arousal and task performance scores. Their findings did not support the hue effect that red is more arousing than blue. Although many advances in recent research have shown, the precise role of the rods and cones of the retina as well as melanopsin in the control of circadian cycle remains to be determined [22]. 


\subsubsection{Psychological Effects and Human Responses to Color}

Research in environmental psychology has demonstrated that different environmental stimuli can affect both mood [3, $23,24]$ and behavior $[3,25,26]$. Previous research suggests a link between colors and emotions, with warm colors associated with aroused feelings and cool colors with calming ones [3, 27, 28]. Tedford, Bergquist, and Flynn [29] investigated the color effects on size in a controlled laboratory setting. They found that when they increased the saturation of a color slide it was more distinct when it was viewed against a gray background. Oyama and Nanri [30] emphasized the role of brightness as a prominent cue in the perception of size of objects regardless of the figure and background color hue contrast. Jacob and Suess [31] investigated the effects of the four primary colors on anxiety states. Forty subjects were asked to state their perceived anxiety levels on a self-report assessment instrument while receiving red, yellow, green, or blue illumination. The results showed that those who were in the red and yellow rooms had significantly higher anxiety scores than those who were in the blue and green rooms. Hemphill [12] examined adults' color and emotion associations and compared them with the findings by Boyatzis and Varghese [32]. Forty subjects were asked to fill out a questionnaire about their favorite color, the major color they were wearing, their emotional feelings to colors, and the reasons for their choices. The researcher found that bright colors were associated with mainly positive emotions and dark colors were associated with negative emotions. Tofle, et al [1] stated that although there are a large number of studies which differentiate between warm and cool colors, a reliable well-evidenced theory that underscores the differences is lacking.

\subsubsection{Individual Perception Differences}

Undoubtedly, individual differences constitute one of the primary reasons behind the incongruity and fragmentation of color research findings. Tofle et al. [1] concluded that "emotional responses to colors are caused by culturally learned associations and by the physiological and psychological makeup of people" (p.5). An explanation for these incongruities effects could be that individuals differ in the way they deal with the environment, how they perceive different aspects of it, and in what ways they process this information. Environmental sensitivity to colors in healthcare settings is likewise another intervening factor. Being hospitalized can increase the feelings of fear, stress and anxiety. These feelings are closely related to the ability to screen out irrelevant stimuli [33], and it could be that being ill results in people having less ability to screen information. As a result, stimulus screening ability may be a concept that varies with people's wellness and may have an essential importance in healthcare settings than in others [3].

\subsubsection{High Screeners/Low Screeners}

Human beings differ in their sensitivity towards environmental stimuli. Mehrabian [34] developed a measure for this environmental sensitivity, which he named Stimulus Screening Ability (SSA). The concept of Stimulus Screening Ability is based on individual differences in arousability as a result of the ability to perceive [3,34]. This research suggests that by effectively screening an environment, the information rate of an environment is reduced, which leads to less arousable responses for high-screeners than for low-screeners of the same environment. This suggests that arousability is inversely related to individual differences in stimulus screening; high-screeners are less arousable persons, and low-screeners are more arousable persons [3, 34].

\subsubsection{Culture, Age, Gender, and Preferences to Color}

Numerous studies have found variations in color preferences across age, gender, and culture, and most literature suggests age differences in color preferences. In addition, recent studies have found that responses to color may vary depending on age, gender, culture, and preference $[4,22]$. Preferences and emotional associations related to a color can be influenced by brightness or saturation [34, 35]; however, saturation has been found to be more important than hue in terms of affecting people's perception of which color is more calming or exciting [14]. Additional variables included types of light sources or background colors [37], the effect of context [35], and cultural factors [37, 38, 39, 40]. Guilford [35] attempted to demonstrate the effects of the three dimensions of color - hue, brightness (tint), and saturation (chroma) - and affective value. He found linear relationships between affective values and both brightness and saturation when hue remained constant. With a constant hue, Guilford [35] concluded that people preferred lighter and more saturated colors to darker and less saturated. With constant brightness and saturation, blue, green, and red were more preferred, and yellow and orange were less preferred. Granger [41] conducted a well-controlled color study to find a general order of color preference and gender effect with fifty subjects representing a wide range of occupations. The research results indicated that the short wavelength colors such as blue and green were preferred to long-wavelength colors such as yellow and red; and the more intense the color, the more preferred. Among many variables, saturation and brightness most strongly influence color preferences. Saturation and brightness determine color preferences within the same hue family; in other words, highly saturated and brighter colors are usually preferred. Dittmar [42] investigated the effect of ageing on color preferences using 842 subjects. For younger and older adults, blue was the most preferred color and yellow was the least preferred. As age increased, the adult preference for blue decreased gradually, whereas the preference for green and red increased. The researcher concluded that adult color preferences change throughout the adult life span. Ellis and Ficek [43], and Silver and Ferrante 
[44indicated that blue was preferred more by adult males than by adult females as the most preferred color; yet blue was still the most preferred color (followed by red) for both genders. It has been observed that males consider the range of cool colors most pleasant, whereas females consider the range of warm colors most pleasant [45]. Culture seems to impact color preferences. While blue was most preferred by Caucasian children in grades 1 through 10 [46], red was selected most often as a favorite color by Mexican children in grades 1 through 7 [37], 11 to 21-year-old full blood Native Americans [46], as well as 6 to 17 -year-old Filipino children [46].

\section{Methods}

Broad Literature review journal articles and reports of empirical studies in multiple disciplines were studied to identify theories, which could have design implications for color in healthcare design. To do so, the peer reviewed journal articles in multiple disciplines including healthcare, medicine, psychology, environmental and interior design, environmental psychology were collected and reviewed.

\subsection{Color in Healthcare Environment}

Healthcare settings are usually spaces of extreme stress for all occupants. Patients, families, and staff experience psychological and physical challenge during diagnostics, procedures, caregiving and care receiving. Recently, potential effects of the physical healthcare environment on the healing and recovery process of patients have been taken into account $[4,47,48]$. Healthcare designers and providers have engaged in practices to promote patients' health, which focuses on reducing stress and increasing pleasantness. Although the contribution of the healthcare environment related to human well-being has not been fully quantified, many researchers have investigated the relationships between the built environment and human health using psychological and physiological indicators of wellness such as measures of stress, mood, productivity, or cognitive performance [49]. Although Dalke et al. [4] and Manke [10] posited that color and light affect patient recovery rates, improving the quality and overall experience of patients, staff and visitors, the research literature of studies about color use in healthcare environments is fragmented and questionable [1]. There is no doubt that healthcare environments should be friendly, therapeutic, and promote healing process but designers need to consider several user groups - the patients, caregivers, visitors, and the community at large. Each of these groups has special functional and perceptual requirements $[1,50]$.

\subsubsection{Reducing Medical Errors}

Medical errors pose serious threats to patient safety [2]. It was estimated that 44,000 to 98,000 people die each year because of medical errors [2]. The scarce literature shows that medical errors are not only caused the mistakes of individuals, but also generated by a combination of space users and environmental conditions. The built environment constituents and approaches have a direct impact on reducing errors [2]. The research conducted at the University of Vienna indicated that room color affects the concentration span of office employees; colored rooms with balanced tension created the greatest concentration [51]. In addition, Ulrich et al, [2] and Buchanan et al [52] clarified that poor lighting levels can also affect the performance of healthcare workers and lead to medical errors.

\subsubsection{Promoting the Sense of Well-being}

Previous studies in healthcare environments support the idea of healing environments where the physical healthcare environment affects the health and well-being of patients [2, 3]. Health outcomes are promoted when physical environments provide a moderate level of positive stimulation; in other words, if stimulation levels are extreme (i.e. too high or too low), the cumulative effect on the patient will be negative on wellness [53]. There is also increasing empirical evidence that poorly designed built environment negatively affects patient well-being [2]. Color and appropriate lighting are useful ways of promoting a sense of well-being and independence [4]. Ulrich et al [2] recommended that the application of color and design for patients' accommodation needs to be taken into consideration to satisfy the emotional and psychological conditions.

\subsubsection{Reducing Stress}

Stress is a process that occurs because of the imbalance between environmental demands and response capabilities of the organism [54, 55]. Zimring [56] pointed that built environment interacts with important behavioral processes which may produce stress for individuals. Additionally, Ulrich et al., [2] indicated that changes in the environment that achieve harm of threaten can cause ill and health problems which emphasizes on the importance of stress process in designed environments. Experiencing stressful situation by patients is an important negative outcome, which directly and adversely affects many other healthcare outcomes. If hospital physical environments contain stressful features or characteristics such as noise, patient stress and other outcomes will often be exacerbated. However, appropriate hospital design that minimizes environmental stressors and fosters exposure to stress-reducing or restorative features could advance improved outcomes. Previous research has claimed that special colors have the ability to arouse people, whereas some others might give people a feeling of calmness [57] which can contribute to reduce stress. Lazarus and Folkman later defined stress as, "a particular relationship between the person and the environment that is appraised by the person as taxing or exceeding his or her resources and endangering his or her well-being" [57]. They view stress as an 
interaction between the person and the environment and this interaction view provides the basis for the ability or inability to deal with a stressful event.

\subsubsection{Improving Patients Sleep \& Circadian Rhythm}

Hospitalized patients have an increased need for sleep because of their illnesses. However, in reality, they often suffer from diminished circadian rhythms and poor sleep while hospitalized, which may lead to increased stress [58], impaired immune function, ventilatory compromise, disrupted thermoregulation, and delirium. These effects may hinder the healing process and contribute to increased morbidity, disorder, and mortality [59, 60]. Mostly all biological functions of the human body in all living organisms vary in roughly 24 - hour cycles. These circadian cycles affect our sleeping/waking, body temperature, eating and drinking habits, mood and many other body mechanisms. Daylight and color have an influential effect on circadian rhythm. Lack of daylight causes the circadian cycles to slow down about 1.1 hours in every 24 hours that causes the releasing of Melatonin at the wrong time and results in delay sleep phase disorder, and dizziness [61]. Pauley [62] indicated that lighting and color should be friendly to the circadian clock of the body. In his point of view it is necessary to consider that the body's need to sleep in darkness that allows the normal production of Melatonin. Previous studies suggest that carefully oriented patient rooms to receive natural daylight and maintain the normal light dark cycle of 24-hour periods, can assist to retain normal circadian rhythms and improve sleep [63]. Although proponents of full-spectrum fluorescent lighting argue that this light source has the advantages of natural light and color for health and performance, the evidences are not enough to support the present claim [62].

\subsubsection{Reducing Length of Stay}

Studies have even reported that an enhanced visual environment has produced improved faster recovery rates by as much as $10 \%$ [4]. Exposure to sunlight has been reported to affect the length of patients' hospital stays [2]. In addition to improvement effects of light and color on recovery according to Smets [64] examinations different colors can affect passage of time which patient feel in hospitals. He asked subjects to estimate the time spent under two different lights, red and blue. The researcher found that those who were under the red light reported shorter estimations than those who were under the blue. She also pointed out that those who with the initial exposure reported shorter time estimations regardless of the light sources.

\subsubsection{Improving Spatial Disorientation \& Way finding}

Wayfinding problems in hospitals are stressful and expensive and have a negative impact on outpatients and visitors, who are unfamiliar with the hospital [2]. Color and appropriate lighting are also powerful tools for coding, navigation and way finding [4]. Recently there are color strategies used in hospitals in order to improve wayfinding and orientation; it can improve the definition of the architectural environment. Color can reinforce the sequences of spaces, landmarks and important features, identifying destinations and differentiating between regions. Appropriate implementing color design can aid intuitive wayfinding, sensory acuity, and cognitive mapping $[4,65]$.

\subsubsection{Increasing Patient Satisfaction}

One study [66] proposed a conceptual model that indicates that physical environments can influence children's health-related outcomes in many ways. They propose that, "this relationship between the physical environment and health outcomes is at least partially mediated by environmental satisfaction and its components" (p. 214).

Environmental satisfaction is a significant predictor of overall satisfaction in healthcare settings [2, 67]. Color as one of the most important components of the physical environment in healthcare settings may be associated with improving patient and staff satisfaction.

\subsubsection{Increasing Staff Morale and Productivity}

From the staff's perspective the environment needed to be helpful to hard work as well as providing places for relaxing for rest periods. Numerous staff stated that a well-designed working environment can aid recruitment and the retention of staff as well as improving morale [2, 4]. The visual environment, including quality of daylight and color, is a vital element influencing hospital staff morale and productivity [4]. According to Hager [52] examinations, by improving the concentration span of employees, appropriate lighting and color design can affect the productivity of staff.

\section{Conclusions}

The findings of the present overview suggest an emphasis on the following themes. First, color is a complicated concept that is intertwined with lighting conditions, individual biases, and the perceived suitability of the color to specific uses. Second, people may rate, perceive, and respond to colors in real settings very differently from tests in laboratories. Third, the meaning of the color itself and the meaning of the colored object may be different in perception processes and it may be questionable to what degree one can find a match between the meaning of a color and a colored object [1]. Fourth, most of previous studies used non-representative samples, others involved with undefined stimulus variables, and some of the conclusions have not been substantiated. Finally, in many of the studies the sample size is often small, and rarely is the research replicated to validate findings. While there is a growing body of literature indicating that color impacts healthcare outcomes by reducing medical errors, promoting the sense of well-being, reducing stress, improving patients sleep, 
reducing length of stay, reducing spatial disorientation, increasing patient satisfaction, and increasing staff morale and productivity (Table 1) - the overall body of knowledge remains fragmented and incongruous [1]. Carefully studying the impacts of color in healthcare settings is complicated and challenging mainly due to the intertwined nature of its various aspects-socio-cultural, psychological, and physiological/biological influences. This is further complicated when taking into account the different types of space users-patients, visitors, medical staff/physicians.

The judgment and perception of healthcare space users exposed to a color in a specific environment are results of a process that is associated with several levels of experiences. For instance, while most of the studies $[1,4,5]$ in the present literature overview addressed partially the person variable $(\mathrm{P})$, the environmental conditions (E) remain unexplored. The person variable subsumes age, gender, origin, culture, education, financial status, which have a direct impact on color perception. The environmental variable (E) includes the interaction between built environment attributes and lighting technical data-spectral energy distribution (SED), color rendering index (CRI), color temperature (CT), and light reflectance value (LRV).

A holistic approach to understanding color perception requires studies that include the interaction and inclusion of the person and environmental conditions simultaneously. The study of color must be intertwined with specific lighting conditions (SED, CRI, and CT) and materials/finishes light reflectance values (LRV). The close relationship between light and color is an important point to be considered. Physically speaking, color and light belong to a single radiant spectrum; without light color cannot be perceived. Therefore, outcomes in healthcare settings are influenced by impact of light and color simultaneously, which necessitates more in depth explorations of the interaction of light and color, but more specifically the impact of light on the perception of color.

Similarly, this complexity is underlined in the fact of patients' inability to screen information. For instance, the same color theme and scheme appears to affect people differently depending on individual characteristics - person variable. However, in healthcare settings the impact of color perception may be of greater complexity, because the reduction of patients' ability to screen out information [1, $3]$.

While previous studies have, on separate basis, partially initiated effervescent lines of inquiry relative to color impact in healthcare environments, the overall body of literature is required to provide sufficient evidences to substantiate that a specific color can influence emotional states or mental and behavioral activities. Future empirical research should be conducted to substantiate concrete evidences. Collaborative research endeavors including but not limited to designers, psychologists and neuro-physicians might lead to innovative and well-evidenced findings.

The advent and emergence of neuro-imaging science namely the use of the functional Magnetic Resonance imaging (fMRI) technology can offer an unparalleled access to biological hard data. This is so important at this juncture, particularly that behavioral data has so far generated incongruous findings. The neuroscience data will provide a methodological platform to support or nullify precedents.

Different studies have considered the role of color in healthcare environment and each of them have looked at different outcomes in the healthcare setting. As the summary of findings, Table 1 shows different studies by researchers on impact of color in healthcare setting.

This topic of study is worthy of further research for three reasons: 1) The healthcare industry is undergoing a boom in the renovation and construction of healthcare facilities, with an estimated $\$ 100$ billion in facilities produced in the United States alone since 2000, 2) the shift in population demographics fueling the baby boomers generation, the first of 78 million baby boomers turned 60 in 2006 and are increasingly reliant on aging healthcare facilities and 3) there is a growing awareness internationally among healthcare administrators and medical professionals of the need to create functional healthcare care environments that have also patient-oriented environments that help patients cope with stress that accompanies stress. Methodologically speaking, it is important to note that most of the overviewed studies $[1,3,4,17]$ explored the color impact from a qualitative perspective; the use of larger sampling - including different countries/cultures and regions, using a psychometric approach might generate more substantiated evidences. Similarly, the inclusion of different gender and age groups might yield more specific findings. In conclusion, currently the use of color in healthcare settings is not based on significant evidence-based empirical studies. It is necessary to note that the attempt to prescribe universal guidelines for appropriate colors in healthcare settings has been unsuccessful. Our efforts should be concentrated on formulating thorough and explanatory theories, and empirical studies with focus on specific and concrete problems rather than abstract and broad questions. 
Table 1. Impact of Color in Healthcare Environments

\begin{tabular}{|c|c|c|c|c|}
\hline $\begin{array}{l}\text { Healthcare } \\
\text { Outcome }\end{array}$ & Issues & Study & Study Design & Findings \\
\hline \multirow{3}{*}{$\begin{array}{c}\text { Reducing } \\
\text { Medical Errors }\end{array}$} & \multirow{3}{*}{$\begin{array}{c}\text { Employees } \\
\text { Concentration \& } \\
\text { Healthcare workers } \\
\text { performance }\end{array}$} & Hager W, (2001) & $\begin{array}{c}45 \text { office employees in Austria were } \\
\text { examined in control and experiment } \\
\text { rooms }\end{array}$ & $\begin{array}{l}\text { Color influenced the concentration } \\
\text { span of office employees. It was } \\
\text { found that grayish rooms produced } \\
\text { less concentration from office staff }\end{array}$ \\
\hline & & Boyce et al., (2008) & $\mathrm{xxx}$ & $\begin{array}{l}\text { Task performance improves with } \\
\text { increased light levels }\end{array}$ \\
\hline & & $\begin{array}{l}\text { Buchanan et al., } \\
\text { (1991) }\end{array}$ & $\begin{array}{c}\text { Direct, undisguised observation } \\
\text { under three levels of illumination on } \\
10,888 \text { prescriptions dispensed by } 5 \\
\text { pharmacies }\end{array}$ & $\begin{array}{l}\text { Less medical error in higher } \\
\text { illumination levels }\end{array}$ \\
\hline \multirow{3}{*}{$\begin{array}{l}\text { Promoting the } \\
\text { sense of } \\
\text { well-being }\end{array}$} & \multirow{3}{*}{$\begin{array}{l}\text { Patient Satisfaction, } \\
\text { Well-being } \\
\text { \& Emotional and } \\
\text { Psychological states }\end{array}$} & $\begin{array}{l}\text { Dijkstra et al., } \\
\qquad(2008)\end{array}$ & $\begin{array}{l}\text { Two studies with } 89 \text { and } 44 \text { subjects } \\
\text { were conducted to This experiment } \\
\text { examined whether individual } \\
\text { differences in environmental } \\
\text { sensitivity influenced emotional } \\
\text { responses (i.e., stress, arousal) and } \\
\text { cognitive appraisal toward different } \\
\text { colors (i.e., green, orange, and } \\
\text { white) in a simulated hospital room. }\end{array}$ & $\begin{array}{l}\text { Orange (pastel) walls had a greater } \\
\text { effect on producing feelings of } \\
\text { arousal than green (pastel) walls had } \\
\text { on reducing stress. Low-screeners } \\
\text { were more affected by the arousal } \\
\text { effects of orange walls and the } \\
\text { stress-reducing affects of green } \\
\text { walls than high-screeners. }\end{array}$ \\
\hline & & Ulrich, et al., (2008) & $\begin{array}{l}\text { Patients from } 20 \text { to } 69 \text { years old who } \\
\text { had undergone cholecystectomy, a } \\
\text { common type of gall bladder } \\
\text { surgery randomly assigned to } \\
\text { different control and experiment } \\
\text { rooms (views to brick walls and } \\
\text { views to trees). } \\
\end{array}$ & $\begin{array}{l}\text { The patients with the tree view had } \\
\text { shorter postoperative hospital stays, } \\
\text { had fewer negative evaluative } \\
\text { comments from nurses, took fewer } \\
\text { moderate and strong analgesic doses, } \\
\text { and had slightly lower scores for } \\
\text { minor postsurgical complications. }\end{array}$ \\
\hline & & Dalke et al., (2006) & $\begin{array}{l}\text { Particular elements of the visual } \\
\text { environment including the use of } \\
\text { appropriate color in interior design, } \\
\text { display of certain types of artwork } \\
\text { and the provision of sunlight and } \\
\text { attractive views out. }\end{array}$ & $\begin{array}{l}\text { Visual elements of the environment } \\
\text { can promote the sense of well-being. }\end{array}$ \\
\hline Reducing Stress & $\begin{array}{l}\text { Arousing and Calming } \\
\text { feelings \& } \\
\text { Sense of well-being }\end{array}$ & $\begin{array}{l}\text { Stone \& English, } \\
\text { (1998) }\end{array}$ & $\begin{array}{l}\text { Students }(n=112) \text { performed either a } \\
\text { low or high demand task in a blue or } \\
\text { red workspace, without or with a } \\
\text { scenic poster. }\end{array}$ & $\begin{array}{l}\text { Perceived task demand was also } \\
\text { marginally related to workplace } \\
\text { color. Satisfaction and performance } \\
\text { were not significantly affected by } \\
\text { posters or workspace color. }\end{array}$ \\
\hline \multirow[t]{2}{*}{$\begin{array}{l}\text { Improving } \\
\text { Patients Sleep \& } \\
\text { Circadian } \\
\text { Rhythm }\end{array}$} & \multirow[t]{2}{*}{$\begin{array}{c}\text { Healing Process, } \\
\text { Patient's sleep quality, } \\
\& \\
\text { Circadian Rhythm }\end{array}$} & $\begin{array}{l}\text { McColl \& Veitch, } \\
\text { (2001) }\end{array}$ & $\begin{array}{l}\text { The impact of lighting design on } \\
\text { sleep quality and healing process }\end{array}$ & $\begin{array}{l}\text { The findings suggest that the proper } \\
\text { use and color of indoor and outdoor } \\
\text { lighting is important to the health of } \\
\text { both humans and ecosystems. } \\
\text { Lighting fixtures should be designed } \\
\text { to minimize interference with } \\
\text { normal circadian rhythms in plants } \\
\text { and animals. }\end{array}$ \\
\hline & & $\begin{array}{l}\text { Wakamura \& } \\
\text { Tokura, (2001) }\end{array}$ & $\begin{array}{l}\text { Seven patients (range } 57-77 \text { years, } \\
\text { males } 3 \text { : females } 4 \text { ) }\end{array}$ & $\begin{array}{l}\text { Bright light exposure during the } \\
\text { daytime affected the induction of } \\
\text { nocturnal deep sleep. }\end{array}$ \\
\hline \multirow{2}{*}{$\begin{array}{l}\text { Improving } \\
\text { Spatial } \\
\text { Disorientation \& } \\
\text { Way finding }\end{array}$} & Intuitive way finding & Evans et al., (1980) & $\begin{array}{c}\text { Role of color coding in } \\
\text { monochromatic environment on } \\
\text { wayfinding behavior }\end{array}$ & $\begin{array}{l}\text { Distinguishing color on accent walls } \\
\text { could be useful in a monochromatic } \\
\text { environment }\end{array}$ \\
\hline & $\begin{array}{l}\text { Memory and } \\
\text { cognition }\end{array}$ & $\begin{array}{l}\text { Goldstein and } \\
\text { Oakley (1986) }\end{array}$ & $\begin{array}{l}\text { Impact of color among individuals } \\
\text { with head-injuries in wayfinding }\end{array}$ & $\begin{array}{l}\text { Color is a more useful aid than form } \\
\text { for individuals with head-injuries } \\
\text { impaired in wayfinding }\end{array}$ \\
\hline $\begin{array}{c}\text { Increasing } \\
\text { Patient } \\
\text { Satisfaction } \\
\end{array}$ & $\begin{array}{l}\text { Positive Mood } \\
\text {, Satisfaction } \\
\text { \& Healing Process } \\
\end{array}$ & $\begin{array}{c}\text { Flynn. J.E. and } \\
\text { Spencer, T. J. (1977) }\end{array}$ & $\begin{array}{l}\text { The Effects of Light Source Color } \\
\text { on User Impression and Satisfaction }\end{array}$ & $\begin{array}{l}\text { Positive impact of white light on } \\
\text { user satisfaction of the users }\end{array}$ \\
\hline $\begin{array}{c}\text { Increasing } \\
\text { concentration } \\
\text { and Productivity } \\
\end{array}$ & Productivity & Paznik (1986) & Impact of light on productivity & $\begin{array}{l}\text { Workers preferred to work in a } \\
\text { lighter setting. This results in more } \\
\text { productivity among workers }\end{array}$ \\
\hline
\end{tabular}




\section{REFERENCES}

[1] R. B. Tofle, B. Shwarz, S. Y. Yoon, and A. Max-Royale, "Color In Healthcare Environments - A Research Report," Coalition for Health Environments Research (CHER), United States2004.

[2] R. S. Ulrich et al., "A Review of the Research Literature on Evidence-Based Healthcare Design," HERD: Health Environments Research \& Design Journal, vol. 1, no. 3, pp. 61-125, 2008.

[3] K. Dijkstra, M. E. Pieterse, and A. T. H. Pruyn, "Individual differences in reactions towards color in simulated healthcare environments: The role of stimulus screening ability," Journal of Environmental Psychology, Article vol. 28 , pp. $268-277,2008$

[4] H. Dalke et al., "Colour and lighting in hospital design," Optics and Laser Technology, Article vol. 38, pp. 343-365, 2006.

[5] K. Nassau, Color for science, art and technology (Azimuth: v. 1). Amsterdam; New York: Elsevier, First edition., 1998.

[6] M. Miller, Color of Interior Architecture. New York: John Wiley \& Sons Inc,, 1997.

[7] E. Verity, Color Observed. Van Nostrand Reinhold, 1980.

[8] J. Pile, Color in Interior Design. McGraw-Hill Education, 1997.

[9] R. Kilmer and W. O. Kilmer, Designing interiors. Hoboken, New Jersey: John Wiley and Sons, Second edition., 2014.

[10] F. Manke, Color, Environment, and Human Response. United States: Wiley, 1996.

[11] F. Birren, Color psychology and color therapy; a factual study of the influence of color on human life. New Hyde Park, N.Y., University Books, 1961.

[12] M. Hemphill, "A note on adults' color-emotion associations," Journal of Genetic Psychology, Article vol. 157, no. 3, p. 275,1996

[13] B. K. Wise and J. A. Wise, "The human factors of color in environmental design: A critical review - NASA-CR-177498 ," ed, 1988.

[14] B. Mikellides., "Color and Physiological Arousal," Journal of Architectural and Planning Research, vol. 7, pp. 13-20, 1990.

[15] A. Bjerstedt, "Warm-cool color preferences as potential personality indicators: Preliminary note," Perceptual and Motor Skills, vol. 10, pp. 31-34, 1960.

[16] S. Rahmatabadi, S. Teimouri and F. Nahidi Azar. "Psychology of Colors and Architectural Façade and Interior Color Selection." Australian Journal of Basic \& Applied Sciences 5, 2011.

[17] K. Engelbrecht, "The Impact of Color on Learning," Perkins \& Will, Chicago, Illinois, 2003
[18] R. Gerard, "Color and emotional arousal". American Psychologist, 13, 340, 1958.

[19] C. W. Erwin, M. Lerner, N. J. Wilson, and W. P. Wilson, "Some further observations on the photically elicited arousal response," Electroencephalography \& Clinical Neurophysiology, vol. 13, pp. 391-394, 1961.

[20] M. R. Ali, "Pattern of EEG recovery under photic stimulation by light of different colors," Electroencephalography \& Clinical Neurophysiology, vol. 33, no. 3, pp. 332-335, 1972.

[21] K. Fehrman and C. Fehrman, Color: the secret influence. Upper Saddle River, N.J.: Prentice Hall, 2000.

[22] Z. O'Connor, "Colour Psychology and Colour Therapy: Caveat Emptor," Color Research and Application, vol. 36, no. 3, pp. 229-234, 2011.

[23] I. Knez, "Effects of Indoor Lighting, Gender, and Age on Mood and Cognitive Performance," Environment \& Behavior, vol. 32, no. 6, p. 817, 11, 2000.

[24] P. Leather, D. Beale, A. Santos, J. Watts, and L. Lee, "Outcomes of environmental appraisal of different hospital waiting areas," Environment and Behavior, vol. 35, no. 6, pp. 842-869, 2003.

[25] R. Gifford, "Light, decor, arousal, comfort and communication," Journal of Environmental Psychology, Article vol. 8, pp. 177-189, 1988.

[26] A. S. Mattila and J. Wirtz, "Congruency of scent and music as a driver of in-store evaluations and behavior," Journal of Retailing, Article vol. 77, pp. 273-289, 2001.

[27] N. Kaya, and H. Epps, "Relationship between Color and Emotion: A Study of College Students." College Student Journal 38, no. 3, 396-405, 2004.

[28] P. Valdez and A. Mehrabian, "Effects of Color on Emotions," Journal of Experimental Psychology: General, vol. 123, no. 4, pp. 394-409, 1994.

[29] W. H. Tedford, S. L. Bergquist, and W. E. Flynn, "The sizecolor illusion," The Journal of general psychology, vol. 97, p. 145, 1977.

[30] T. Oyama, "Synthesis of perceptual information on hue, brightness, shape, size and spatial position," The Japanese Journal of Psychonomic Science, vol. 22, no. 1, pp. 108-114, 2003.

[31] K. W. Jacobs and J. F. Suess, "Effects of four psychological primary colors on anxiety state," Perceptual and Motor Skills, vol. 41, no. 1, pp. 207-210, 1975.

[32] C. J. Boyatzis and R. Varghese, "Children's emotional associations with colors," Journal of Genetic Psychology, Article vol. 155, no. 1, p. 77, 1994

[33] A. Mehrabian, "Theory and Evidence Bearing on a Scale of Trait Arousability," Current Psychology, vol. 14, no. 1, pp. 3-28, 1995.

[34] A. Mehrabian, "A questionnaire measure of individual differences in stimulus screening and associated differences in arousability," Environmental Psychology \& Nonverbal Behavior, vol. 1, no. 2, pp. 89-103, 1977. 
[35] J. P. Guilford, "The affective value of color as a function of hue, tint, and chroma," Journal of Experimental Psychology, vol. 17, no. 3, pp. 342-370, 1934.

[36] L. Sivik, "Color Meaning and Perceptual Color Dimensions." Goteborg Psychological Reports 4, no. 1, 1974.

[37] I. Gesche, "The color preferences of one thousand one hundred and fifty-two Mexican children," Journal of Comparative Psychology, vol. 7, no. 4, pp. 297-311, 1927.

[38] A. Choungourian, "Color preferences and cultural variation," Perceptual And Motor Skills, vol. 26, no. 3, pp. 1203-1206, 1968.

[39] S. Wiegersma and A. van Loon, "Some variables in the blue (red) phenomenon," The Journal of General Psychology, vol. 116 , no. 3, p. $259,1989$.

[40] M. Saito, "A comparative study of color preferences in Japan, China and Indonesia, with emphasis on the preference for white," Perceptual and Motor Skills, vol. 83, no. 1, pp. 115-128, 1996.

[41] G. W. Granger, "An Experimental Study of Colour Preferences," Journal of General Psychology, vol. 52, no. 1, p. 3, 01, 1955.

[42] M. Dittmar, "Changing colour preferences with ageing: A comparative study on younger and older native Germans aged 19-90 years," Geronotology, vol. 47, no. 4, pp. 219-226, 2001.

[43] L. Ellis and C. Ficek, "Color preferences according to gender and sexual orientation," Personality and Individual Differences, vol. 31, no. 8, pp. 1375-1379, 2001.

[44] N. C. Silver and R. Ferrante, "Sex differences in color preferences among an elderly sample," Perceptual and Motor Skills, vol. 80, no. 3, Pt 1, pp. 920-922, 1995.

[45] H. Helson and T. G. Lansford, "The role of spectral energy of source and background color in the pleasantness of object colors," Applied Optics, vol. 9, no. 7, pp. 1513-1562, 1970.

[46] T. R. Garth and I. R. Collado, "The color preferences of Filipino children," Journal of Comparative Psychology, vol. 9, no. 6, pp. 397-404, 1929.

[47] A. S. Devlin and A. B. Arneill, "Health Care Environments and Patient Outcomes: A Review of the Literature," Environment \& Behavior, Article vol. 35, no. 5, pp. 665-694, 2003.

[48] M. Schweitzer, L. Gilpin, and S. Frampton, "Healing Spaces: Elements of Environmental Design That Make an Impact on Health," Journal of Alternative \& Complementary Medicine, Article vol. 10, pp. S-71-S-83, 2004.

[49] J. G. P. Park and C. Park, "Color Perception in Pediatric Patient Room Design: American versus Korean Pediatric Patients," Health Environments Research \& Design Journal (HERD) (Vendome Group LLC), Article vol. 6, no. 4, pp. 10-26, 2013.

[50] W. Ruga, "Designing for the six senses," Journal Of Health Care Interior Design: Proceedings From The ... Annual National Symposium On Health Care Interior Design.
National Symposium On Health Care Interior Design (U.S.), vol. 1, pp. 29-34, 1989.

[51] T. L. Buchanan, K. N. Barker, J. T. Gibson, B. C. Jiang, and R. E. Pearson, "Illumination and errors in dispensing," American Journal Of Hospital Pharmacy, vol. 48, no. 10, pp. 2137-2145, 1991.

[52] W. Hager, "Colour and Room." Wiesner-Hager Mobel GmbH, 2001.

[53] J. F. Wohlwill, "The Physical Environment: A Problem for a Psychology of Stimulation," Journal of Social Issues, vol. 22, no. 4, pp. 29-38, 1966.

[54] R. S. Lazarus and S. Folkman, Stress, appraisal, and coping. New York: Springer Pub. Co., 1984.

[55] R. S. Lazarus, Psychological stress and the coping process (McGraw-Hill series in psychology). New York, McGraw-Hill, 1966.

[56] C. Zimring, "The Built Environment as a Source of Psychological Stress: Impacts of Buildings and Cities on Satisfaction and Behavior," Environmental Stress, 1984.

[57] N. J. Stone, and A.J English. "Task Type, Posters, and Work Space Color on Mood, Satisfaction, and Performance." Journal of Environmental Psychology 18, 175-85, 1988.

[58] M. A. F. P. Novaes, A. Aronovich, M. B. Ferraz, and E. Knobel, "Stressors in ICU: patients' evaluation," Intensive Care Medicine, Author abstract no. 12, p. 1282, 1997.

[59] S. L. Krachman, G. E. D'Alonzo, and G. J. Criner. Sleep in the intensive care unit. 1995.

[60] S. Parthasarathy and M. Tobin, "Sleep in the intensive care unit," Intensive Care Med, vol. 30, no. 2, pp. 197-206, 2004.

[61] B. Matusiak, "Daylight Design for Healthy Learning Environments." Professional lighting design magazine 73, 48-52, 2010.

[62] S. M. Pauley, "Lighting for the human circadian clock: recent research indicates that lighting has become a public health issue," Medical Hypotheses, Article vol. 63, pp. 588-596, 2004.

[63] T. Wakamura and H. Tokura, "Influence of bright light during daytime on sleep parameters in hospitalized elderly patients," Journal of physiological anthropology and applied human science, vol. 20, no. 6, p. 345, 2001.

[64] G. Smets, "Time Expression of Red and Blue." Perceptual and Motor Skills 29, 511-14, 1969.

[65] K. Lynch, Good city form. Cambridge, Mass.: MIT Press, 1984, c1981. 1st paperback ed., 1984.

[66] A. S. Sandra, S. Mardelle McCuskey, and W. V. James. (2005) Children's Environments and Health-Related Quality of Life: Evidence Informing Pediatric Healthcare Environmental Design. [research article]. 186.

[67] P. B. Harris, C. Ross, G. McBride, and L. Curtis, "A place to heal: environmental sources of satisfaction among hospital patients," Journal of Applied Social Psychology, Abstract no. 6, p. 1276, 2002. 